\title{
Quantum-size effects in the energy loss of charged particles interacting with a confined two-dimensional electron gas
}

\author{
A. G. Borisov, ${ }^{1,2}$ J. I. Juaristi, ${ }^{3,4}$ R. Díez Muiño, ${ }^{2,4}$ D. Sánchez-Portal, ${ }^{2,4}$ and P. M. Echenique ${ }^{2,3,4}$ \\ ${ }^{1}$ Laboratoire des Collisions Atomiques et Moléculaires (CNRS UMR 8625), Bâtiment 351, Université Paris-Sud, \\ 91405 Orsay Cedex, France \\ ${ }^{2}$ Donostia International Physics Center DIPC, P. Manuel de Lardizabal 4, 20018 San Sebastián, Spain \\ ${ }^{3}$ Departamento de Física de Materiales, Facultad de Químicas UPV/EHU, Apartado 1072, 20080 San Sebastián, Spain \\ ${ }^{4}$ Unidad de Física de Materiales, Centro Mixto CSIC-UPV/EHU, P. Manuel de Lardizabal 3, 20018 San Sebastián, Spain
}

(Received 28 July 2005; published 17 January 2006)

\begin{abstract}
Time-dependent density-functional theory is used to calculate quantum-size effects in the energy loss of antiprotons interacting with a confined two-dimensional electron gas. The antiprotons follow a trajectory normal to jellium circular clusters of variable size, crossing every cluster at its geometrical center. Analysis of the characteristic time scales that define the process is made. For high-enough velocities, the interaction time between the projectile and the target electrons is shorter than the time needed for the density excitation to travel along the cluster. The finite-size object then behaves as an infinite system, and no quantum-size effects appear in the energy loss. For small velocities, the discretization of levels in the cluster plays a role and the energy loss does depend on the system size. A comparison to results obtained using linear theory of screening is made, and the relative contributions of electron-hole pair and plasmon excitations to the total energy loss are analyzed. This comparison also allows us to show the importance of a nonlinear treatment of the screening in the interaction process.
\end{abstract}

DOI: 10.1103/PhysRevA.73.012901

PACS number(s): 34.50.Bw, 71.10.Ca, 71.15.Mb, 78.70.- $\mathrm{g}$

\section{INTRODUCTION}

A fundamental aspect of the interaction of particles with matter is the excitation of target electrons, leading to energy loss by the moving projectile. From the theoretical point of view, a large amount of work has been devoted in the past to study these processes, both from quantum-mechanical and semiclassical points of view [1]. As a consequence, a profound understanding of the underlying physics has been reached. Most of the existing approaches are based on the many-body response of the target to the external perturbation, as calculated in the frequency domain. Such approximation provides direct information on the final states of the system after the interaction, but the time dynamics does not appear explicitly. Moreover, calculations based on the linearresponse approximation fail to describe the target excitation in the case of strong perturbations, and nonperturbative approaches are required [2].

Growing interest in ultrafast processes triggered by the development of the femto- and attosecond lasers and related techniques [3] naturally puts forward a question on how the electronic excitation of the system develops in time [4,5]. It has been shown, for example, that the screening response, one of the basic properties of electron media, needs finite time to be established [6-9]. Furthermore, for nanosized targets, the interaction time between the projectile and the target electrons is, in many situations, much shorter than the characteristic time needed for the density excitation produced in the target to explore the whole system $[8,9]$. The many-body excited modes of the nano-object are then not resolved. From the point of view of the moving projectile, the finite-size object would behave on the corresponding time scale as an infinite system so that there will be no quantum-size effects on the projectile stopping. In other words, if the spectrum of the perturbation is broad enough to overlap many of the discrete excited states of the system, the continuum limit can be retrieved. The shorter the perturbation is, the broader is its spectrum in the frequency domain, and therefore, less sensitive is the response to the boundary conditions [10].

Time-dependent density-functional theory (TDDFT) is particularly well suited to address the questions outlined above because it provides a self-consistent nonperturbative time-domain treatment of the dynamics of the many-body system in response to the external (strong) perturbation [11-13]. Here, we report on the TDDFT study of the energy loss of antiprotons penetrating a two-dimensional (2D) electron gas confined into circular jellium clusters of variable size. Only energy loss of the projectile due to electronic excitations in the cluster is considered. We thus do not treat the nuclear stopping. The antiproton follows a trajectory normal to the cluster surface and crosses the cluster at its geometrical center. In this respect, our work differs from previously published studies, where in-plane, or parallel to the 2D electron gas plane, trajectories were considered [14-18].

Quasi-two-dimensional electronic media appear in a variety of systems of interest, such as image states [19], surface states [20], metal overlayers on metals [21] and semiconductors [22], semiconductor heterostructures [23], and quantum dots [24]. In the present case, the use of a pure 2D electron gas approximation implies that the model is, strictly speaking, restricted to the projectile velocity range where the (quantized) states in the direction of the projectile motion (perpendicular to the 2D electron gas plane) cannot be excited. Typical values for the excitation energies of perpendicular modes are of few electron volts for metal overlayers 
and surface states, and of the order of tenths of electron volts in semiconductor quantum dots and heterostructures. If the lateral size of the object is much larger than the size in the perpendicular direction, energy band gaps due to quantization in the perpendicular direction will be much larger than those associated to the lateral dimensions. Thus, one can always find a low-velocity regime in which the perpendicular modes cannot be excited and finite size effects in the projectile energy loss will primarily depend on quantization in the lateral direction. The latter constitutes the subject of the present contribution.

We will show that the time at which electronic excitations are produced is rather well defined and closely corresponds to the moment at which the projectile crosses the cluster plane. Hence, the particular geometry used here allows an extended discussion on the different time scales of importance for the process: (i) the interaction time defined by the projectile velocity and (ii) the time on which the excited modes of the finite-size system are established. If the former time is essentially shorter than the latter, the finite-size effects disappear. This happens even for quite slow collisions, provided that the lateral extension of the system is large enough. Nevertheless, we will present results for velocities of up to few atomic units, where the excitation of the perpendicular modes cannot be neglected and the model used here is no longer able to represent realistic quasi-2D systems. These results are included just for illustrative purposes, to stress the role of the finite-interaction time effects.

The paper is organized as follows: the theoretical background and the numerical details of our TDDFT calculations are provided in Sec. II; results of these calculations are discussed in Sec. III; similar results obtained using linear theory of screening are presented in Sec. IV to analyze the process in further detail; and the main conclusions of our work are summarized in Sec. V. Atomic units $\left(e=m_{e}=\hbar=1\right)$ are used unless otherwise stated.

\section{TDDFT CALCULATION OF THE ENERGY LOSS}

As outlined in the introduction we study the energy lost by an antiproton colliding with a 2D cluster (jellium disk), made of a constant background of positive charge with density $n_{+}(\rho)$, plus a fixed number of electrons that make the system neutral. We call $\rho$ to the radial coordinate in the $2 \mathrm{D}$ plane. We use closed-shell clusters of variable radius $R_{c}$, comprising from 16 to 16000 electrons, $N_{e}$. The positive background density is described by the parameter $r_{s}: n_{+}(\rho)$ $=1 / \pi r_{s}^{2}$, if $\rho \leqslant R_{c l}$, and $n_{+}(\rho)=0$ for $R_{c}<\rho$. The number of electrons in the cluster is given by $N_{e}=\left(R_{c l} / r_{s}\right)^{2}$. Calculations presented in this work are performed with $r_{s}=2$. The projectile follows the classical trajectory $Z_{a}(t)=-Z_{0}+v t$ along the $z$ axis chosen perpendicular to the plane of the cluster (located at $z=0$ ) and going through the center of the cluster. To simplify the analysis of the results, the velocity $v$ is assumed to be constant during the collision. This approximation can be easily relaxed, and the projectile trajectory can be defined from the Ehrenfest molecular-dynamics approach $[25,26]$, which is similar to the recent work on the hydrogen interaction with small 3D jellium clusters by Baer and Siam [13].
However, within the velocity range considered here, the constant velocity approximation holds with good precision as follows from the comparison between the calculated energy losses and the total energy of the projectile.

The time evolution of the electronic density $n(\vec{r}, t)$ in response to the field of the projectile is calculated with TDDFT. We work within the Kohn-Sham (KS) scheme solving the time-dependent 2D KS equations:

$$
i \frac{\partial \Psi_{j}(\vec{r}, t)}{\partial t}=\left\{T+V_{\mathrm{eff}}(\vec{r}, t)\right\} \Psi_{j}(\vec{r}, t)
$$

where $\Psi_{j}(\vec{r}, t)$ are the KS orbitals, $T$ is the kinetic energy operator, and $V_{\text {eff }}(\vec{r}, t)$ is the effective potential. Initial conditions $\Psi_{j}(\vec{r}, t=0)$ are given by the Kohn-Sham orbitals of the unperturbed system (jellium cluster). The numerical procedure used here is similar to that reported previously in Ref. [9]. We work in cylindrical coordinates $(\rho, \phi, z)$ well adapted to the axial symmetry of the system. Recalling that the $2 \mathrm{D}$ electron gas is confined to the plane, located at $z=0$, the KS orbitals can be expressed as

$$
\Psi_{j}(\vec{r}, t)=\psi_{j}(\rho, t) \frac{e^{i m_{j} \phi}}{\sqrt{2 \pi}},
$$

where $m$ is a good quantum number and it is preserved in due course along the time propagation. Since $\pm m$ states are degenerate, in practice we only consider the time evolution of the $\mathrm{KS}$ orbitals corresponding to $m \geqslant 0$. The timedependent electronic density $n(\rho, t)$ is then obtained as a sum over occupied KS states

$$
n(\rho, t)=\sum_{j \in \mathrm{occ}} s_{j}\left|\psi_{j}(\rho, t)\right|^{2}
$$

where $s_{j}$ accounts for the spin and $m$ degeneracy $\left(s_{j}=2\right.$ for $m=0$, and $s_{j}=4$ otherwise). As shown previously [27], for the finite-difference representation of the kinetic-energy operator, efficiency of the time-propagation can be largely improved if the variable change is introduced in the spatial coordinate $\rho$ in order to densify the grid close to the origin of coordinates. Therefore, we use $\rho=f(x)$, where $f(x)=x$ $-b \arctan (x / \sqrt{a}) / \sqrt{a}, a=500$ and $b=490$ being parameters. A uniform mesh is used in $x$ variable: $x_{k}=x_{0}+\Delta x(k-1)$, with the number of knots ranging from 1200 to 1800 , depending on the cluster size. The spacing is $\Delta x=0.1$ for the smaller clusters and $\Delta x=0.2$ for the clusters with $N_{e}=4328$ and $N_{e}=16022$. The variable change is accompanied by the following change of the wave function:

$$
\psi_{j}(x, t)=\frac{\varphi_{j}(x, t)}{\left[f(x) f^{\prime}(x)\right]^{1 / 2}}
$$

where $f^{\prime} \equiv d f / d x$. The time-evolution of the $\varphi_{j}(x, t) \mathrm{KS}$ orbitals is then given by the time-dependent $\mathrm{KS}$ equation [see Eq. (1)], with the kinetic-energy operator possessing explicitly symmetric form 


$$
T \rightarrow T\left(m_{j}\right)=-\frac{1}{2} \frac{1}{\left(f f^{\prime}\right)^{1 / 2}} \frac{d}{d x} \frac{f}{f^{\prime}} \frac{d}{d x} \frac{1}{\left(f f^{\prime}\right)^{1 / 2}}+\frac{m_{j}^{2}}{2 f^{2}} .
$$

Note that $T$ explicitly depends on the KS state $j$ through the centrifugal energy.

The effective potential $V_{\text {eff }}(\rho, t)$ comprises several terms

$$
V_{\mathrm{eff}}(x, t)=V_{\mathrm{ext}}(x, t)+V_{\mathrm{es}}(x, t)+V_{\mathrm{xc}}(x, t)
$$

where $V_{\text {ext }}(x, t)=\left[Z_{a}(t)^{2}+\rho^{2}\right]^{-1 / 2}=\left[Z_{a}(t)^{2}+f(x)^{2}\right]^{-1 / 2}$ is the external potential created by the negative point charge. $V_{\mathrm{es}}(x, t)$ is the electrostatic potential. $V_{\mathrm{xc}}(x, t)$ is the exchange-correlation potential calculated within the adiabatic local-density approximation (LDA), using the parametrization of the 2D exchange-correlation energy given by Tanatar and Ceperley [28]. In difference with Ref. [9], no optical-absorbing potential $[29,30]$ was introduced at the boundary of the radial mesh. Indeed, absorption of the electrons possibly emitted into continuum as a result of the antiproton impact would lead to the nonconservation of the number of particles and thus precludes a converged calculation of the total electronic energy of the system at the end of the collision, as well as of the force acting on the projectile. We have explicitly checked that for the size of the $\rho$ mesh used here $\left(0 \leqslant \rho \leqslant R_{c l}+65\right)$, eventual reflection of the emitted electrons at the grid boundaries (quantization of the freeelectron continuum) does not affect the final results.

The action of the kinetic-energy operator [Eq. (5)] is calculated using three-point finite difference [27]. With the Hamiltonian operator projected on the grid, the KS orbitals of the unperturbed system are obtained by direct diagonalization. The time propagation is done with the split-operator technique [31,32], separating the potential- and kineticenergy terms

$$
\begin{aligned}
\varphi_{j}(x, t+\Delta t)= & e^{-i(\Delta t / 2) V_{\text {eff }}[x, t+(\Delta t / 2)]} e^{-i \Delta t T\left(m_{j}\right)} \\
& \times e^{-i(\Delta t / 2) V_{\text {eff }}[x, t+(\Delta t / 2)]} \varphi_{j}(x, t) .
\end{aligned}
$$

The action of the exponential with the kinetic-energy operator is calculated employing Cayley transform [33]

$$
e^{-i \Delta t T\left(m_{j}\right)}=\frac{1-i \frac{\Delta t}{2} T\left(m_{j}\right)}{1+i \frac{\Delta t}{2} T\left(m_{j}\right)}+O\left(\Delta t^{3}\right)
$$

leading to the three-diagonal system of linear equations connecting $\varphi_{j}\left(x_{k}, t+\Delta t\right)$ and $\varphi_{j}\left(x_{k}, t\right)$ at the grid points. A typical time step used in the calculation is $\Delta t=0.002$ a.u. The detailed discussion on the implementation of the wave-packet propagation in cylindrical coordinates can be found in Ref. [27].

For the electrostatic potential calculations, the following procedure is used. The electrostatic potential created by the 2D circular charge distribution is given by

$$
\begin{aligned}
V_{\mathrm{es}}(\rho, t) & \equiv V_{\mathrm{es}}\left(\rho ;\left[n(\rho, t)-n_{+}\right]\right) \\
& =2 \pi \int_{0}^{\infty} d q J_{0}(q \rho) \int_{0}^{\infty} \rho^{\prime} d \rho^{\prime} J_{0}\left(q \rho^{\prime}\right)\left[n\left(\rho^{\prime}, t\right)-n_{+}\left(\rho^{\prime}\right)\right],
\end{aligned}
$$

where $J_{0}(q \rho)$ is the Bessel function. The discrete form of Eq. (9) reads

$$
V_{k}(t)=\sum_{k^{\prime}} \mathcal{R}_{k k^{\prime}}\left[n_{k^{\prime}}(t)-n_{+k^{\prime}}\right]
$$

where, taking into account the variable change, $V_{k}(t)$ $\equiv V_{\mathrm{es}}\left(x_{k},\left[n_{k}(t)-n_{+k}\right]\right)$, and $n_{k}(t)-n_{+k} \equiv n\left(x_{k}, t\right)-n_{+}\left(x_{k}\right)$. The matrix $\mathcal{R}_{k k^{\prime}}$ is given by

$$
\mathcal{R}_{k k^{\prime}}=2 \pi \frac{\Delta x}{f\left(x_{>}\right)} \frac{1}{\Gamma(1)} F\left[\frac{1}{2}, \frac{1}{2}, 1,\left(\frac{f\left(x_{<}\right)}{f\left(x_{>}\right)}\right)^{2}\right] f\left(x_{k^{\prime}}\right)\left(\frac{d f}{d x}\right)_{x=x_{k^{\prime}}} .
$$

$F$ is the hypergeometric function, $x_{>}\left(x_{<}\right)$stands for the largest (smallest) of $x_{k}$ and $x_{k^{\prime}}$, and $\Delta x$ is the step in the $x$ mesh. In defining Eq. (11), we have used the following identity:

$$
\int_{0}^{\infty} d q J_{0}(q \rho) J_{0}\left(q \rho^{\prime}\right)=\frac{1}{\rho_{>}} \frac{1}{\Gamma(1)} F\left[\frac{1}{2}, \frac{1}{2}, 1,\left(\frac{\rho_{<}}{\rho_{>}}\right)^{2}\right],
$$

where $\rho_{>}\left(\rho_{<}\right)$is the largest (smallest) of $\rho$ and $\rho^{\prime}$. The logarithmic divergence of $\mathcal{R}_{k k^{\prime}}$ for $x_{k}=x_{k^{\prime}}$ can be avoided calculating the potential in the midpoints $V_{k+1 / 2}(t)$ and then interpolating to the points of the mesh.

From the time-dependent electronic density, we obtain the force acting on the antiproton

$$
F_{z}(t)=2 \pi \int_{0}^{\infty} f f^{\prime} d x \frac{n(x, t)-n_{+}(x)}{\left(Z_{a}(t)^{2}+f^{2}\right)^{3 / 2}} Z_{a}(t)
$$

and the final energy loss suffered by the projectile

$$
\Delta E=\left|v \int_{0}^{\infty} F_{z}(t) d t\right| .
$$

The quality of the calculation is tested by comparing the energy loss $\Delta E$ and the change of the total energy of the cluster, given by $\Delta \mathcal{W}=\mathcal{W}(t=\infty)-\mathcal{W}(t=0)$, where

$$
\begin{aligned}
\mathcal{W}(t)= & \sum_{j \in \mathrm{occ}} s_{j} 2 \pi \int_{0}^{\infty} d x \varphi_{j}^{*}(x, t) T\left(m_{j}\right) \varphi_{j}(x, t) \\
& +\pi \int_{0}^{\infty} f f^{\prime} d x V_{\mathrm{es}}(x ;[n(x, t)]) n(x, t) \\
& -2 \pi \int_{0}^{\infty} f f^{\prime} d x V_{\mathrm{es}}\left(x ;\left[n_{+}\right]\right) n(x, t) \\
& +2 \pi \int_{0}^{\infty} f f^{\prime} d x E_{x c}[n] n(x, t),
\end{aligned}
$$

where $E_{x c}[n]$ is the 2D exchange-correlation energy given in parametrical form by Tanatar and Ceperley [28]. Observe 


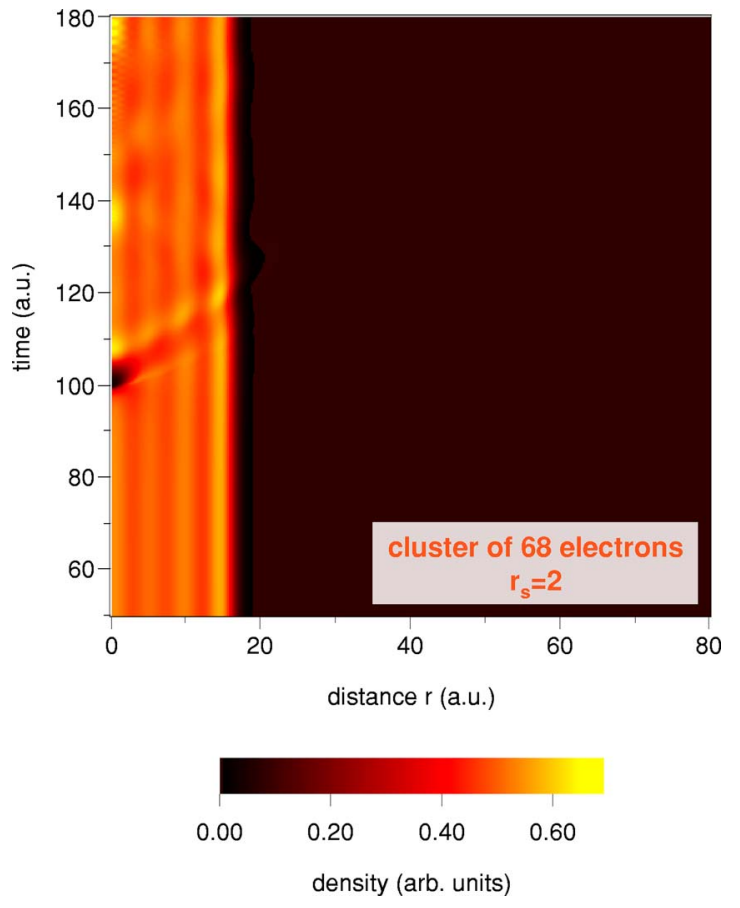

FIG. 1. (Color online) Interpolated image of the time evolution of the electronic density in a two-dimensional jellium cluster. The horizontal axis represents the distance from the center of the cluster $r$ (in atomic units), and the vertical axis represents the time $t$ (in atomic units). The electronic density is modified in time by the interaction with an antiproton, whose trajectory is perpendicular to the cluster and crosses it precisely at its center $(r=0)$. The velocity of the antiproton is $v=1$ a.u. The cluster is made of $N_{e}=68$ electrons and the electronic density parameter is $r_{s}=2$. The radius of the cluster is thus $R_{c}=N_{e}^{1 / 2} r_{s} \approx 16.5$ a.u. Arbitrary units are used for the electronic density. Color codes used for the plot are shown in the inset.

that since we are interested in the $t \rightarrow \infty$ limit, the interaction of the projectile with the positive background and the electrons is not accounted for in the total energy calculation. Typically, $\Delta E$ and $\Delta \mathcal{W}$ quantities are equal within a fraction of a percent. However, for the systematic study of the energy losses, the scheme given by Eqs. (13) and (14) is preferable. It only depends on an integral over the density, and thus the numerical convergence is much easier to reach than that of $\Delta \mathcal{W}$, the latter containing derivatives of KS orbitals.

\section{TDDFT RESULTS}

We start with a general description of the time evolution of the electronic density when the confined 2D system is perturbed by a moving antiproton. As an example, we show in Figs. 1 and 2 the total electronic density in the jellium cluster as a function of time, when the incident antiproton, moving with a trajectory perpendicular to the cluster, crosses it at its center. The velocity of the antiproton is $v=1$ a.u., and the electronic-density parameter in both clusters is $r_{s}=2$. Initially, the antiproton is located 100 a.u. away from the cluster $\left(Z_{0}=100\right.$ a.u.). Figure 1 shows the case of a relatively small cluster, with $N_{e}=68$ electrons and radius

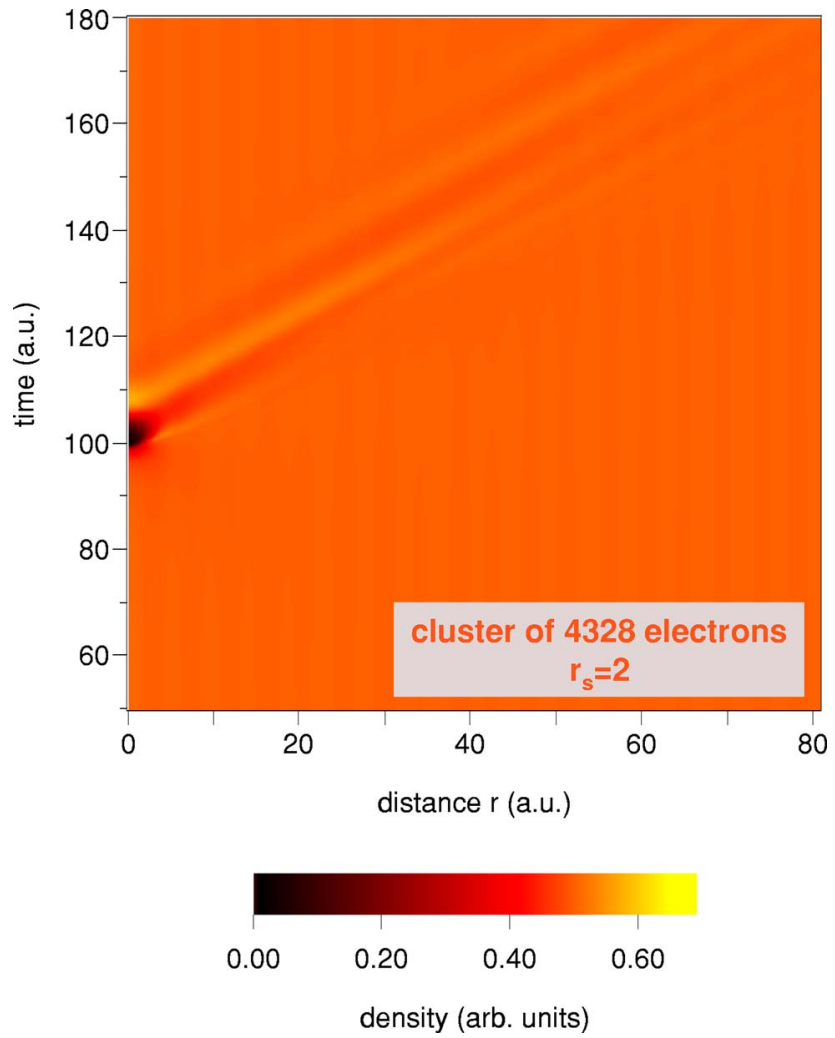

FIG. 2. (Color online) The same as in Fig. 1, but for a jellium cluster of $N_{e}=4328$ electrons. The radius of the cluster is thus $R_{c}=N_{e}^{1 / 2} r_{s} \approx 131.6$ a.u.

$R_{c}=N_{e}^{1 / 2} r_{s} \approx 16.5$ a.u. Figure 2 shows the case of a bigger cluster, with $N_{e}=4328$ electrons and $R_{c} \approx 131.6$ a.u. Hence, the border of the cluster is clearly visible in Fig. 1 but is out of the range of the plot in Fig. 2. The impact time at which the antiproton hits the system distinctly appears in both plots as a dark spot around the center of the cluster $(\rho=0)$ at a time of $t \approx 100 \mathrm{a}$.u. The Coulomb repulsion between the antiproton and the cluster electrons forces the latter out of the cluster center, creating a hole of electronic charge. At this particular instant of time, the perturbation created in the cluster is highly local and the electronic density away from the center remains basically unaffected for both clusters.

Once the antiproton leaves the system and is placed at a distance sufficiently large, the screening hole becomes a net charge that attracts back the electrons. Oscillations in the electronic density of the cluster are thus created in the process of smearing out the local unbalance of charge. The timedependent evolution of the screening in a $2 \mathrm{D}$ system in response to a sudden perturbation was previously studied in detail [9]. In a 2D free-electron gas, the dispersion of the 2D plasmon modes in the long wavelength limit $(k \rightarrow 0)$ is given by $\omega=\sqrt{2 k} / r_{s}$, with $k$ being the wave vector. As a result, collective oscillations are rapidly damped because of the dephasing of different modes, and the local electronic density quickly reaches the unperturbed limit. The disturbance of the 2D electron gas by a sudden perturbation leads to a solitary shock wave of electronic density that travels along the system with velocity close to the Fermi one $\left(v_{F}=\sqrt{2} / r_{s}\right)$. The density ahead and behind the wave front does not de- 


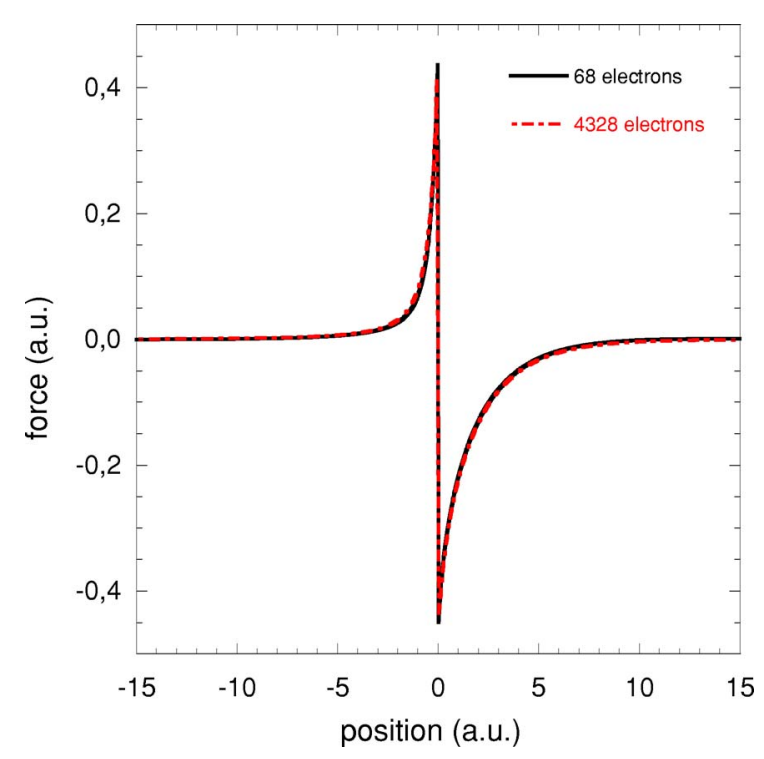

FIG. 3. (Color online) Theoretical calculation of the force on the projectile for an antiproton following a trajectory perpendicular to a two-dimensional jellium cluster and crossing it precisely at its center. The force is plotted as a function of the antiproton position with respect to the cluster. The antiproton velocity is $v=1$. Results for two different clusters are shown: a cluster with $N_{e}=68$ electrons [solid (black) line], and a cluster with $N_{e}=4328$ electrons [dashed (red) line). The cluster electronic density is defined in both cases by $r_{s}=2$. All quantities in atomic units.

pend on time (see Figs. 1 and 2). This picture breaks down when the density wave hits the cluster boundaries and is reflected back, i.e., when finite-size effects appear. For the time scale shown in the figures, the interference pattern is only present in the case of the smallest cluster: the reflection of the electronic density wave at the borders of the cluster results in an interference pattern with several bright spots at $\rho=0$, which represent the periodic appearance of piling up of charge at the cluster origin. It is worth mentioning that the energy transfer from the projectile to the cluster electrons is related to the asymmetry in the time evolution of the electronic density with respect to the impact time. Quantitative information on the stopping process can be obtained from the force.

The force felt by the moving antiproton due to the interaction with the jellium cluster [Eq. (13)] is plotted in Fig. 3 as a function of the antiproton position, and for the same two clusters. The antiproton crosses the cluster at $z=0$. The velocity of the antiproton is again $v=1$ a.u. Hence, the horizontal axis also represents a measurement of time (referred to the crossing instant) in atomic units. In previous literature, the force has been described as a sum of two contributions, namely, a nondissipative component and a dissipative component [34]. These two contributions could be associated to the real and imaginary parts of the incident particle selfenergy, respectively. A theoretical treatment, such as ours, does not allow to discriminate between these two components. Separation of the two contributions from the knowledge of the ground-state potential-energy surface [13] would not work in the present case, in which the final state corresponds to a highly excited cluster.
The force on the moving particle only takes appreciable values close to the crossing instant and is very similar for both cluster sizes. At 10 a.u. of distance away from the cluster (or, equivalently, 10 a.u. of time before and after crossing the cluster), the force has nearly vanished and the interaction process is over. Energy transfer between the moving particle and the confined system takes place in this restricted interval of time. Figures 1 and 2 show that in this time interval, the change in electronic density induced by the antiproton in its passage has not reached the borders of the cluster yet. Otherwise said, for these particular clusters and this particular value of the velocity, the interaction process is over before the induced electronic density could be affected by the presence of the cluster borders. This explains the almost identical force produced on the antiproton by clusters with such different number of electrons.

Another important feature of the force is that it has different sign for the antiproton in its way in and in its way out. In its approach to the cluster, the antiproton feels an attractive force derived from the cluster polarization. On its way out, the antiproton is also attracted by the excited cluster left behind, but now the force acts in a direction opposite to that of the antiproton movement. In particular, the screening hole left behind by the antiproton contributes to the projectile stopping. The total energy loss suffered by the antiproton arises from the asymmetry of the force at the two sides of the cluster.

We show in Fig. 4 the energy lost by an antiproton as a function of its velocity, for several cluster sizes and keeping the electronic density parameter $r_{s}=2$ fixed. The cluster left behind by the antiproton is a system in an excited state. The amount of energy finally transferred in the process is the one left in the cluster when the antiproton is already far away from it. The electronic energy gained by the jellium cluster is balanced by the loss in kinetic energy of the moving antiproton. For velocities larger than $v=0.3$ a.u., the energy loss is very similar for all system sizes. For instance, at a velocity close to the energy loss peak $(v=1$ a.u. $)$, there is roughly a $5 \%$ difference in the energy lost by the antiproton for systems with such huge differences in size, as a cluster of $N_{e}=16$ electrons $\left(R_{c}=8\right.$ a.u. $)$ and a cluster of $N_{e}=16022$ electrons $\left(R_{c} \approx 253\right.$ a.u. $)$. Although details of the time evolution of the electronic energy differ from one cluster to another, the total energy transferred to the cluster at infinite time is very similar in all cases. Locality in time and space of the energy-transfer process prevents the appearance of finitesize effects. The final value of the energy lost by the antiproton is thus determined by the local properties of the jellium cluster, which are similar in all cases. Local properties of the system are thus the only ones tested by the incident particle for the high-velocity range. A calculation of the energy loss for the infinite 2D electron gas performed using linear theory (see Sec. IV) is also shown in the plot. The linear-theory calculation merges into the cluster calculation for high velocities, precisely at its range of validity.

Conditions at which the locality of the energy-loss process breaks down and finite-size effects start to appear depend on the particle velocity and on the particular sistem size. As the velocity decreases, the deviation of the energy loss from the common curve first starts for smaller clusters. 


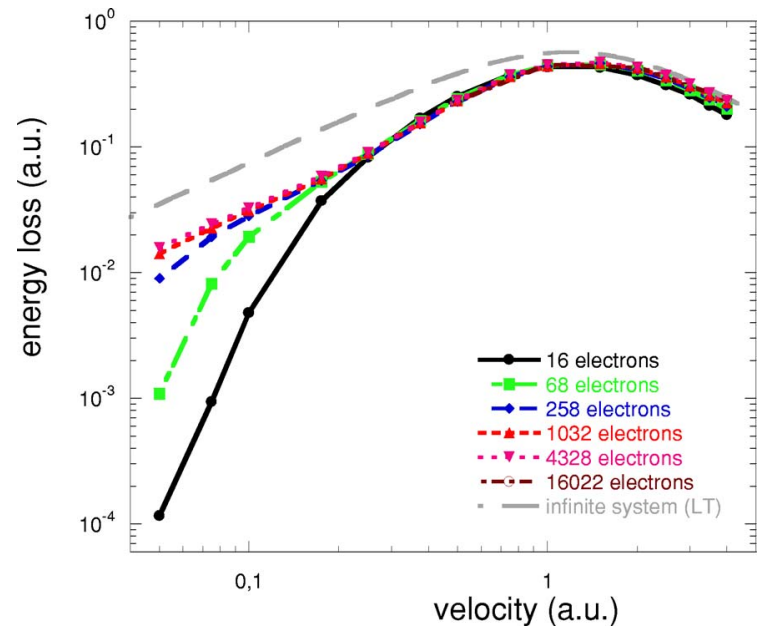

FIG. 4. (Color online) Energy lost by an antiproton moving with a trajectory perpendicular to a two-dimensional circular jellium cluster. The antiproton crosses the cluster precisely at its center. The energy loss is plotted as a function of the antiproton velocity. Results for clusters with different number of electrons $N_{e}$ are shown: $N_{e}=16$ electrons (black circles), $N_{e}=68$ electrons (green squares), $N_{e}=258$ electrons (blue diamonds), $N_{e}=1032$ electrons (red up-triangles), $N_{e}=4328$ electrons (pink down-triangles), and $N_{e}=16022$ electrons (brown open circles). Lines connecting the marks are also shown to guide the eye (solid, long-dashed, dashed, short-dashed, dotted, and dash-dotted, respectively). The cluster electronic density is defined in all cases by $r_{s}=2$. The energy loss for an antiproton crossing perpendiculary an infinite twodimensional electron gas, as calculated in linear theory, is shown as well by a gray long-dashed line, without marks. All quantities in atomic units.

This deviation is due to the discretization in energy of the cluster excited states. Slow antiprotons are less effective in creating electronic excitations in the cluster when the energy difference between states is too large. The typical energy difference between levels in a cluster $\Delta \epsilon$ depends on its size and is larger for smaller clusters, due to quantum confinement conditions. The geometry of the problem, a particle with a trajectory perpendicular to a finite $2 \mathrm{D}$ system, allows one to define, in a simple way, an interaction time between the antiproton and the jellium cluster. The force on the antiproton presents non-negligible values for a path $L$ of few tens of atomic units. Typical interaction times $\tau$ between the antiproton and the jellium cluster are thus of the order of $\tau \sim L / v$, and the width of the spectrum of the time-dependent perturbation is $\omega \sim 1 / \tau \sim v / L$. In a simplified picture, if the difference between energy levels in the cluster $\Delta \epsilon$ is bigger than this energy $\Delta \epsilon>v / L$, excitations cannot be created and the antiproton energy loss is suppressed.

A time-dependent picture can also be used to understand the effect. The density excitation needs a certain time $\tau_{e}$ to explore the finite-size cluster. For a 2D cluster of radius $R_{c}$, $\tau_{e}=2 R_{c} / v_{e}$, where $v_{e}$ is the characteristic velocity, close to the Fermi one. For fast projectiles and the particular trajectory considered in our problem, $\tau_{e} \gg \tau$ and the energy loss is independent of $R_{c}$. For slower projectiles, $\tau_{e} \sim \tau$, and the energy loss depends on the value of $R_{c}$. In the limit $\tau_{e} \ll \tau$, the target electrons have enough time to rearrange themselves in the presence of the projectile and the process falls into the adiabatic regime, with no energy loss associated.

\section{LINEAR-THEORY CALCULATION OF THE ENERGY LOSS}

In order to gain more information about both finite-size effects and nonlinearities, we have performed calculations based on linear-response theory taking as a target an infinite $2 \mathrm{D}$ jellium. In this approach, the energy change per unit time suffered by an external particle can be calculated in terms of the force overcome by the particle due to the induced potential it creates. We define as positive the energy transferred to the target. In linear-response theory, the change in energy per unit time is as follows:

$$
\frac{d E}{d t}=\mathbf{v} \int d^{3} \mathbf{r} n_{\mathrm{ext}}(\mathbf{r}, t) \nabla V_{\mathrm{ind}}(\mathbf{r}, t) .
$$

In our case, the external charge is a negative unit charge $\left(Z_{1}=-1\right)$ traveling perpendicular to the infinite $2 \mathrm{D}$ jellium

$$
n_{\mathrm{ext}}(\mathbf{r}, t)=Z_{1} \delta(z-v t) \delta(\mathbf{R}),
$$

where $z$ is the coordinate perpendicular to the target $(z=0$ the position of the target) and $\mathbf{R}$ corresponds to parallel coordinates. By writing the induced potential $V_{\text {ind }}$ in terms of the dielectric-response function of the 2D infinite medium $[\epsilon(Q, \omega)]$, we obtain the following expressions:

$$
\begin{aligned}
\frac{d E}{d t}= & -\frac{2 Z_{1}^{2} v}{\pi} \int_{0}^{\infty} d Q Q e^{2 Q v t} \int_{0}^{\infty} d \omega \frac{\omega}{\omega^{2}+Q^{2} v^{2}} \\
& \times \operatorname{Im}\left\{-\frac{1}{\epsilon(Q, \omega)}\right\}, \quad t<0 \\
\frac{d E}{d t}= & \frac{2 Z_{1}^{2} v}{\pi} \int_{0}^{\infty} d Q Q e^{-2 Q v t} \int_{0}^{\infty} d \omega \frac{\omega}{\omega^{2}+Q^{2} v^{2}} \operatorname{Im}\left\{-\frac{1}{\epsilon(Q, \omega)}\right\} \\
+ & \frac{4 Z_{1}^{2} v^{2}}{\pi} \int_{0}^{\infty} d Q Q^{2} e^{-Q v t} \int d \omega \frac{\sin (\omega t)}{\omega^{2}+Q^{2} v^{2}} \\
& \times \operatorname{Im}\left\{-\frac{1}{\epsilon(Q, \omega)}\right\}, \quad t>0 .
\end{aligned}
$$

The first term on the right-hand side of Eq. (19) is identical, although opposite in sign, to the right-hand side of Eq. (18). Therefore, the only contribution to the total energy loss for the entire path traveled by the particle can be obtained by considering only the second term in Eq. (19).

The energy lost by the antiproton up to a given instant of time $t_{0}$ can be obtained by integrating Eqs. (18) and (19) from $t=-\infty$ up to $t=t_{0}$

$$
\begin{aligned}
\Delta E\left(t_{0}\right)= & -\frac{Z_{1}^{2}}{\pi} \int_{0}^{\infty} d Q e^{2 Q v t_{0}} \int_{0}^{\infty} d \omega \frac{\omega}{\omega^{2}+Q^{2} v^{2}} \\
& \times \operatorname{Im}\left\{-\frac{1}{\epsilon(Q, \omega)}\right\}, \quad t_{0}<0,
\end{aligned}
$$




$$
\begin{aligned}
\Delta E\left(t_{0}\right)= & -\frac{Z_{1}^{2}}{\pi} \int_{0}^{\infty} d Q e^{-2 Q v t_{0}} \int_{0}^{\infty} d \omega \frac{\omega}{\omega^{2}+Q^{2} v^{2}} \\
& \times \operatorname{Im}\left\{-\frac{1}{\epsilon(Q, \omega)}\right\}+\frac{4 Z_{1}^{2} v^{2}}{\pi} \int_{0}^{\infty} d Q Q^{2} \int_{0}^{\infty} d \omega \\
& \times \frac{1}{\left(\omega^{2}+Q^{2} v^{2}\right)^{2}}\left(\omega-e^{-Q v t_{0}\left[\omega \cos \left(\omega t_{0}\right)\right.}\right. \\
& \left.\left.+Q v \sin \left(\omega t_{0}\right)\right] \operatorname{Im}\left\{-\frac{1}{\epsilon(Q, \omega)}\right\}\right), \quad t_{0}>0 .
\end{aligned}
$$

The total energy loss in the entire path is obtained by evaluating Eq. (21) in the limit $t_{0} \rightarrow \infty$

$$
\Delta E=\frac{4 Z_{1}^{2} v^{2}}{\pi} \int_{0}^{\infty} d Q Q^{2} \int_{0}^{\infty} d \omega \frac{\omega}{\left(\omega^{2}+Q^{2} v^{2}\right)^{2}} \operatorname{Im}\left\{-\frac{1}{\epsilon(Q, \omega)}\right\} .
$$

As explained above, this result can also be obtained by integrating only the second term of Eq. (19) from $t=0$ to $\infty$.

In this work, we use the dielectric response derived by Stern in the random phase approximation (RPA) [35]. In this approach, the excitations contributing to $\operatorname{Im}\{1 / \epsilon(Q, \omega)\}$ include two different kind of processes: single particle (electron-hole pair creation) and collective (plasmon) excitations. The regions in the $(Q, \omega)$ plane for these two kinds of excitations are well separated. The electron-hole formation occurs for those $(Q, \omega)$ values for which $\operatorname{Im}\{1 / \epsilon(Q, \omega)\} \neq 0$. This condition is verified in the region limited by the curves $\omega=-Q Q_{F}+Q^{2} / 2$ and $\omega=Q Q_{F}+Q^{2} / 2$, where $Q_{F}=(2 \pi n)^{1 / 2}$ is the Fermi wave vector for a $2 \mathrm{D}$ electron gas of density $n$. The plasmon excitation is characterized by the condition $\epsilon(Q, \omega)=0$, which defines a single curve $w_{p}(Q)$ in the $(Q, \omega)$ plane. For small values of $Q$, the plasmon frequency behaves as $\omega_{p}^{2}(Q) \simeq Q_{F}^{2} Q$. These conditions allow one to disentangle the contribution of single-particle and plasmon excitations to the different terms that characterize the energy loss of the particle.

The validity and limitations of the linear-theory approach can be extracted from Fig. 4. At high velocities, for which the antiproton can be considered as a small perturbation, the agreement obtained within the results of linear theory and the nonlinear calculation is very good. Since, as discussed above, finite-size effects are not important in this range of velocities, the infinite medium approximation is not an important shortcoming in this case. The importance of using a nonlinear approach is observed in the low-velocity sector of this figure. Even in the case of the largest clusters considered, for which an infinite medium approach should be a reliable approximation, linear-theory results overestimate the total energy loss. The overestimation (underestimation) of the stopping power for slow negative (positive) singly charged particles traveling in bulk matter, is a well-known shortcoming of linear response, which considers the projectile as a small perturbation. The difference between our nonlinear results for large clusters and those obtained within linear response in the low-velocity regime, allows one to quantify this effect for the problem that we are treating.

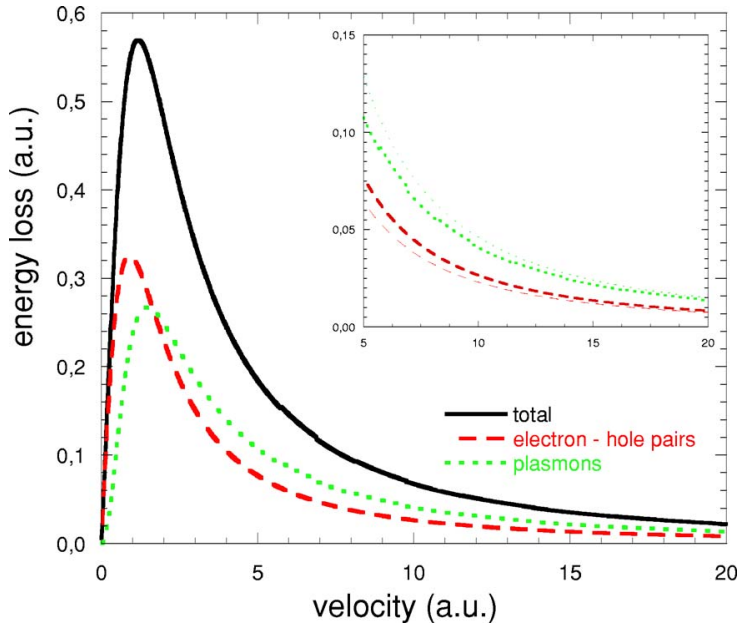

FIG. 5. (Color online) Energy loss suffered by an antiproton crossing a two-dimensional electron gas as a function of its velocity. The calculation is performed using linear response theory and a RPA response function. The electronic density is defined by $r_{s}=2$. The solid (black) line is the total energy loss, the dashed (red) line is the contribution of electron-hole pair excitations, and the dotted (green) line is the contribution of plasmon excitations. The inset shows the calculated contributions of the electron-hole pairs and the plasmon excitations, using two different models for the response function: thick lines correspond to the RPA calculation and thin lines to the high-velocity limit of the plasmon-pole response function. Line codes are identical to those in the main plot. All quantities in atomic units.

In Fig. 5, we show the total energy loss suffered by the particle in its entire path, as a function of its velocity, as calculated within linear-response theory. We separate the contributions coming from the excitation of plasmons and electron-hole pairs. In the low-velocity regime, the energy lost by the projectile is mainly devoted to the creation of electron-hole pairs, whereas at high velocities contributions coming from both kinds of excitations are important, though more energy is transferred to the plasmon field.

In order to understand better the separation of the transferred energy among plasmons and electron-hole pairs in the high-velocity regime, let us take as an example a plasmonpole approximation for the imaginary part of the inverse of the response function. This approximation allows us to perform the integrals of Eq. (22), analytically,

$$
\begin{aligned}
\operatorname{Im}\left\{-\frac{1}{\epsilon(Q, \omega)}\right\}= & \frac{\pi}{2} \omega_{p}(Q) \delta\left[\omega-\omega_{p}(Q)\right] \Theta\left(Q_{c}-Q\right) \\
& +\frac{\pi}{2} \frac{\omega_{p}^{2}(Q)}{Q^{2} / 2} \delta\left(\omega-\frac{Q^{2}}{2}\right) \Theta\left(Q-Q_{c}\right) .
\end{aligned}
$$

The first term corresponds to the plasmon contribution and the second one to electron-hole excitations. We approximate the plasmon frequency by its long wavelength limit $\left[\omega_{p}(Q)\right.$ $\left.=Q_{F} Q^{1 / 2}\right], \Theta(x)$ is the Heaviside step function, and $Q_{c}$ is the value of the wave vector for which the plasmon frequency cuts the electron-hole excitation energy $\left[\omega_{p}\left(Q_{c}\right)=Q_{c}^{2} / 2\right]$. In- 
troducing Eq. (23) into Eq. (22) and taking the limit of high velocities, we get the following for the plasmon $\left(\Delta E_{p l}\right)$ and electron-hole $\left(\Delta E_{e-h}\right)$ contributions to the energy loss:

$$
\begin{gathered}
\Delta E_{p l}=\frac{4 Z_{1}^{2} Q_{F}^{2}}{v^{2}} \ln (v), \\
\Delta E_{e-h}=\frac{2 Z_{1}^{2} Q_{F}^{2}}{v^{2}} \ln (v) .
\end{gathered}
$$

This shows that, within this simple approximation to the response function, the plasmon contribution to the energy loss is twice that of electron-hole pairs in the high-velocity regime. The values obtained using Eqs. (24) and (25) are also represented in the inset of Fig. 5 and constitute a good approximation to the exact RPA results at high velocities. Nevertheless, the ratio obtained at high velocities in the RPA case is somewhat smaller than two. Finally, let us mention that the ratio that we find between the partial contributions to the energy loss is not a general property. In the case of a particle traveling through a three-dimensional electron gas, the energy loss at high velocities is equally shared between plasmons and electron-hole pairs [36]. Bret and Deutsch [14] showed that for a fast particle traveling inside a $2 \mathrm{D}$ electron gas, the energy loss is dominated by electron-hole pairs. The precise separation of the energy loss among the different channels obtained in our case is a specific property of the geometry of our problem.

\section{CONCLUSIONS}

In summary, the study of energy-loss processes in the time domain offers a complementary perspective to the standard frequency-domain approach. In particular, we have theoretically studied the energy loss suffered by an antiproton that crosses a 2D electron gas of finite size, following a trajectory perpendicular to the system plane. The geometry of our problem allows us to define an effective interaction time for the process. Comparison of this interaction time to the characteristic times in which the electronic excitations in the system develop and reach the system boundaries prescribes two different regimes for the energy loss. For the systems considered in this work and relatively high velocities of the moving antiproton (typically $v>0.3$ a.u.), the interaction between the projectile and the finite-size object is over before the density wave created by the projectile can be reflected by the boundaries. This implies that the energy spectrum of the perturbation is broad enough to overlap many of the excited states of the system. In this regime the continuum limit of the infinite system is retrieved. The energy loss mainly depends on the local electronic properties of the system, and the dependence on the system size is not significant. For smaller velocities, the interaction time between the projectile and the system increases, the energy spectrum of the perturbation becomes narrower, and the discretization of levels in the finite system plays a role. In the time domain, quantum-size effects in the energy loss due to electronic excitations can be predicted to appear whenever the interaction time between the projectile and the target is comparable to the time required for the perturbed electronic density to be significantly affected by the presence of system boundaries. In this velocity range and for realistic systems, interaction of the projectile with the target nuclei may also play a role and contribute to the total energy loss.

Another interesting conclusion from the present work concerns the possibility of projectile trapping. As follows from the results presented in Fig. 4 for the large-enough system, the energy loss is linear with projectile velocity. At the same time, the projectile energy is given by the parabolic law. Therefore, there is a certain velocity range for which the initial energy of the projectile is smaller than the energy loss expected for the given velocity. Studies performed using the Ehrenfest molecular dynamics approach $[25,26]$ for a test projectile of effective mass $m_{\text {eff }}=1$ show that slow-enough projectiles can be trapped by the 2D electron gas. Similar results were reported for the hydrogen atom interacting with a small $\left(N_{e}=19\right)$ 3D jellium cluster [13]. The projectile is first trapped into an oscillatory trajectory. Then, depending on the size of the system, either the projectile is continuously slowed down till landing at the $z=0$ plane (infinite system) or the amplitude of the oscillations around the $z=0$ plane is progressively reduced until it is stabilized at a given value, when the motion is too slow to further excite the system (finite cluster).

\section{ACKNOWLEDGMENTS}

This work was supported, in part, by the Basque Departamento de Educación, Universidades e Investigación, the University of the Basque Country UPV/EHU (Grant No. 9/UPV 00206.215-13639/2001), the Spanish Ministerio de Educación y Ciencia (Grant No. FIS2004-06490-C03-00), and the EU Network of Excellence NANOQUANTA (Grant No. NMP4-CT-2004-500198). A.G.B. acknowledges financial support by the DIPC.
[1] P. M. Echenique, F. Flores, and R. H. Ritchie, Solid State Phys. 43, 229 (1990); A. Sarasola, R. H. Ritchie, E. Zaremba, and P. M. Echenique, Adv. Quantum Chem. 46, 1 (2004).

[2] P. M. Echenique, R. M. Nieminen, and R. H. Ritchie, Solid State Commun. 37, 779 (1981).

[3] A. Föhlisch, P. Feulner, F. Hennies, A. Fink, D. Menzel, D. Sanchez-Portal, P. M. Echenique, and W. Wurth, Nature (Lon- don) 436, 373 (2005).

[4] R. Huber, F. Tauser, A. Brodschelm, M. Bichler, G. Abstreiter, A. Leitenstorfer, Nature (London) 414, 286 (2001).

[5] L. Bányai, Q. T. Vu, B. Mieck, and H. Haug, Phys. Rev. Lett. 81, 882 (2001).

[6] G. S. Canright, Phys. Rev. B 38, 1647 (1988).

[7] M. Alducin, J. I. Juaristi, and P. M. Echenique Surf. Sci. 559, 
233 (2004).

[8] A. Borisov, D. Sánchez-Portal, R. Díez Muiño, and P. M. Echenique, Chem. Phys. Lett. 387, 95 (2004).

[9] A. Borisov, D. Sánchez-Portal, R. Díez Muiño, and P. M. Echenique, Chem. Phys. Lett. 393, 132 (2004).

[10] R. Peierls, Surprises in Theoretical Physics (Princeton University Press, Princeton, 1979), pp. 64-67.

[11] F. Calvayrac, P. G. Reinhard, E. Suraud, and C. A. Ullrich, Phys. Rep. 337, 493 (2000).

[12] A. Castro, M. A. L. Marques, J. A. Alonso, and A. Rubio, J. Comput. Theor. Nanosci. 1, 230 (2004).

[13] R. Baer and N. Siam, J. Chem. Phys. 121, 6341 (2004)

[14] A. Bret and C. Deutsch, Phys. Rev. E 48, 2994 (1993).

[15] You-Nian Wang and Teng-Cai Ma, Phys. Rev. A 55, 2087 (1997).

[16] A. Bergara, I. Nagy, and P. M. Echenique, Phys. Rev. B 55, 12864 (1997)

[17] E. Zaremba, I. Nagy, and P. M. Echenique, Phys. Rev. Lett. 90, 046801 (2003).

[18] E. Zaremba, I. Nagy, and P. M. Echenique, Phys. Rev. B 71, 125323 (2005).

[19] P. M. Echenique and J. B. Pendry, J. Phys. C 11, 2065 (1978).

[20] P. M. Echenique, R. Berndt, E. V. Chulkov, Th. Fauster, A. Goldmann, and U. Höfer, Surf. Sci. Rep. 52, 219 (2004).

[21] E. V. Chulkov, J. Kliewer, R. Berndt, V. M. Silkin, B. Hellsing, S. Crampin, and P. M. Echenique, Phys. Rev. B 68, 195422 (2003).

[22] E. Rotenberg, H. Koh, K. Rossnagel, H. W. Yeom, J. Schäfer,
B. Krenzer, M. P. Rocha, and S. D. Kevan, Phys. Rev. Lett. 91, 246404 (2003).

[23] H. Haug and A. P. Jauho, Quantum Kinetics in Transport and Optics of Semiconductors (Springer, New York, 1996).

[24] S. M. Reimann and M. Manninen, Rev. Mod. Phys. 74, 1283 (2002).

[25] P. Ehrenfest, Z. Phys. 45, 455 (1927).

[26] D. Marx and J. Hutter, Ab Initio Molecular Dynamics: Theory and Implementation (Modern Methods and Algorithms of Quantum Chemistry, Proceedings), 2nd ed., edited by J. Grotendorst NIC Series Vol. 3, (John von Neumann Institute for Computing, Julich, 2000), pp. 329-477.

[27] A. G. Borisov, J. P. Gauyacq, and S. V. Shabanov, Surf. Sci. 487, 243 (2001).

[28] B. Tanatar and D. M. Ceperley, Phys. Rev. B 39, 5005 (1989).

[29] D. Neuhauser and M. Baer, J. Chem. Phys. 91, 4651 (1989).

[30] U. V. Riss and H.-D. Meyer, J. Chem. Phys. 105, 1409 (1996).

[31] M. D. Feit and J. A. Fleck, Jr., J. Chem. Phys. 78, 301 (1982).

[32] M. D. Feit, J. A. Fleck, Jr., and A. Steiger, J. Comput. Phys. 47, 412 (1982).

[33] W. H. Press, B. P. Flannery, S. A. Teukolsky, and W. T. Vetterling, Numerical Recipes (Cambridge University Press, Cambridge, England, 1989).

[34] P. M. Echenique, R. H. Ritchie, N. Barberán, and J. Inkson, Phys. Rev. B 23, 6486 (1981).

[35] F. Stern, Phys. Rev. Lett. 18, 546 (1967).

[36] J. K. Lindhard, K. Dan. Vidensk. Selsk. Mat. Fys. Medd. 28(8), 1 (1954). 